\title{
ENABLING INTELLIGENT AGENTS AND THE SEMANTIC WEB FOR E-COMMERCE
}

\author{
Yefim Kats \\ Assistant Professor in Computer Information Systems \\ Institute of Technology, State University of New York \\ P.O. Box 3050, Utica, New York 13504-3050, USA \\ e-mail: katsy@sunyit.edu
}

\begin{abstract}
This paper examines an impact of the growing intelligent agent technologies and the Semantic Web on the phenomenon of e-commerce. We discuss the problems-technical as well as legal-arising from the emergence of the new forms of intelligent software and consider the possible solutions. In particular, we review how the integration of the Semantic Web and intelligent agents can provide a new environment for the secure and scalable e-commerce applications
\end{abstract}

Keywords: Intelligent Agents, Software Engineering,E-Commerce, Web Ontologies, Semantic Web

\section{INTRODUCTION}

In this paper we explicate some of the issues related to the emerging forms of intelligent software, primarily in respect to e-commerce and the newly arriving phenomenon of the Semantic Web. In these areas intelligent software in general and intelligent agents in particular offer significant benefits while posing special challenges for the information technology professionals as well as the users.

The range of relevant problems is very wide and covers not only technical but also legal issues related to e-Commerce, Semantic Web, Web Ontologies, and intelligent agent technologies. Needless to say, the paper is not conceived as a comprehensive tutorial but rather as an outline of the growing problems and possible solutions.

\section{INTELLIGENT AGENTS AND E-COMMERCE}

The essential feature of e-commerce is the technical ability to conduct electronic transactions including credit card processing, bartering etc. However, the availability of technical infrastructure is not sufficient for a successful implementation of a successful business model. Many businesses of the 'new economy' era quickly disappeared, because their creators discounted the human factors involved in launching and effectively implementing a Web based business model.
In particular, 'new economy' companies apparently did not take into consideration the increasing interplay between the technological and the social components in the effective and efficient implementation of the networked global business enterprise. The inefficient customer service represents just one example of the 'bottleneck' for the otherwise viable startup companies. This bottleneck may be due not only to the managerial failure, but also to the technical inability to link heterogeneous business environments, the lack of security standards etc. Another (and related) example is the shopping cart technology. About $75 \%$ of on-line customers abandon their shopping carts. The reasons are manifold, including semantic mismatch between the global partners, inefficient customer support, security and privacy related concerns as well as inefficient data and knowledge management.

The newly emerging artificial intelligence technology - intelligent agents - is socially oriented and, thus, could become an important component able to fill the gap between the technological and social dimensions of a modern Web based enterprise. Of course, this process is coupled with the development of new business oriented protocols and the new ways to process semantically rich content.

The benefits of intelligent agents are two-fold technical and commercial. The commercial benefits are based on their ability to support a specific type 
of customer behavior. The generic types of buying behavior pattern are valid for all e-commerce models; the buying behavior proceeds through the following typical stages:

- Need identification

- Product brokering

- Merchant brokering

- Negotiation

- Purchase

- Product service

- Follow-up evaluation of customer satisfaction

Examples of the artificial agents assisting customers to carry out aforementioned tasks include [1]:

- Monitors and notification agents - useful for need identification

- PersonaLogic and Tête-à-Tête - enabled to assist in product brokering

- BargainFinder and Jango - used for the merchant brokering phase

- AuctionBot and Kasbah provide help in the negotiation stage of a transaction

The patterns of customer behavior determine not only the benefits but also the possible damage a particular agent technology can cause and, consequently, the types of constraints to be imposed on the corresponding agents. In this context, the benefits and the side effects of agent technologies are closely interrelated. For example, the need identification procedures can be - and often are excessively invasive. The intelligent technologies are enabled and often intended to amplify the potentially abusive impact of the intrusive business practices. In this respect, the developers of such technologies should be able to distinguish between a support tool design and a 'social engineering' tool design; to that effect an appropriate set of constraints should be embedded into the existing as well as emerging agent design methodologies.

These considerations are especially relevant to the so-called mobile intelligent agents representing a form of distributed computing. Such agents are particularly useful for product brokering, merchant brokering, and negotiation. The technical benefits provided by mobile agents include $[2,3,4]$ :

- Parallel processing

- Efficient use of system resources

- Reduced network load

- Ability to make dynamic decisions

- High level of autonomy
In spite of obvious advantages, mobile agents pose new challenges both technical and legal [2, 3, 4]. In a most trivial sense, mobile agents act in a way similar to certain viruses and may cause similar problems. The fact that they execute on the server side entails special security risks such as unauthorized data access, data destruction, and abuse of host machine resources.

In the world of e-commerce the type of 'agent abuse' depends on the agent's business profile. For example, negotiating agents may renege on their obligation to complete a transaction once they encounter a more promising opportunity. This type of problem could be addressed at the Web services protocol level. We could label the desired property as a capacity for atomic negotiation - ones a particular business transaction completed it cannot be rolled back. What particular Web service protocol (or a combination of protocols) to be used is an open question. We should mention, in this context, the so called Business Transaction Protocol (BTP) developed by the OASIS Business Transactions Technical Committee [5].

The agent's misbehavior may be either intentional or simply arising from the fact that the ecommerce agents typically work in a semantically uncertain environment. In order to control the problems related to uncertainty, the special metrics need to be developed, capable of capturing the uncertainty of e-commerce transactions. The possible solution could involve using fuzzy logic or, possibly, certain algebraic structures able to capture such notions as identity, similarity etc.

The recent litigation process involving data collection from eBay server shows very subtle technical and legal problems associated with the use of robots on the Web. It was admitted during the court hearing that robots accessed the site approximately 100000 times a day, though it accounted for a little more than $1 \%$ of all requests received by eBay. The court admitted nevertheless that permission to crawl eBay site could result in reduced system performance, system unavailability and data loss [6].

The problems related to functionality of intelligent agents in a distributed environment extend beyond the issues raised by eBay litigation, considering that online auction represents only one business model among many; other models such as brokerage or storefront present new technical and legal challenges. Consequently, the viable solution calls for the development of the multifaceted framework for the virtual reality of e-commerce. In this context, the functionality of intelligent business applications should be addressed at several levels: 
- Operational requirements aimed at optimization of the distributed business applications

- Social/legal requirements related to the data collection techniques, and to agent-to-agent and agent-to-human interactions

- Software engineering requirements

- Requirements related to the 'semantics' of e-commerce environment

The required measures should incorporate a number of technical, business, and legal issues including:

- Development of the legal framework and the appropriate technical as well as business protocols for Web agents

- Development of agent enabled semantic infrastructure

- Development of agent oriented software engineering tools

The software engineering aspect of agent technologies is a key to any meaningful expansion of global e-commerce infrastructure. In the next section we discuss the emerging agent oriented software engineering methodologies and the popular intelligent agents' development platforms.

\section{SOFTWARE ENGINEERING OF AGENTS AND MULTIAGENT SYSTEMS}

Generally speaking, we can distinguish between the two equally important aspects of agent oriented software engineering: first, the development of viable design methodologies for agents and multiagent systems, and second, the ability to use agents themselves as CASE (Computer Aided Software Engineering) tools. The development of agent based CASE tools would help to fill the gap between the rapid progress in software functionality on the one hand, and the slow progress in the software engineering area on the other hand. In particular, the newly discovered security flaws in all types of software (including almost all releases of Windows OS) may be, at least partly, attributed to the disparity between the growing size and complexity of software packages vs. the outdated methods of software development. A number of methodologies focused on building agents and multiagent systems have been already developed; some of the most popular approaches include:

- GAIA - based on object oriented approach and applicable to multiagent systems
- MaSE - multiagent systems engineering methodology

- AOR - agent-object relationship approach

- AUML - agent unified modeling language

On the whole, the 'large-scale methodologies like GAIA or MaSE are somewhat restrictive and do not suite well the multiagent interactions in the heterogeneous Web environment. An approach represented by the AUML, an 'agent-oriented' extension of the Unified Modeling Language (UML), is more narrowly focused and based on the most often quoted aspect of agent technology: intelligent agents are, to large extent, active subjects typically operating within the agent community. Conceived initially as a generic agent-oriented modeling language, the AUML is also well positioned to accommodate the requirements of the Web based commerce. Among the proposed ecommerce oriented AUML features are triggers, atomic transactions, and reliable connectivity [7].

The agent oriented software engineering led to the development of proprietary as well as free source agent development platforms; some of the currently popular platforms include:

- JADE LEAP - implemented in JAVA by LEAP consortium, supports multiagent systems

- JACK - Belief, Desire, and Intent agent systems (BDI); based on JAL - JAVA extension

- Agent Factory - integrated BDI oriented framework (open source)

- Agent Builder - an integrated development tool

JAVA, as a general purpose software development platform, is also agent enabled, in particular, due to aglets - movable JAVA objects. Further efforts needed to ensure that integrated JAVA Web services development platform such as J2EE fully accommodates agent technologies with all necessary security constraints. In fact, security mechanisms, embedded into the competing platforms such as Java J2EE or .NET, provide only a limited answer to the concerns of business community. In this context, it is important to realize that the growth of mobile intelligent agent technologies has been contributing to the rapid erosion of the traditional distinction between distributed and local applications, an issue especially significant for business oriented software.

Moreover, Java based and other platforms must become not only security enabled but also the Semantic Web enabled. This assumes the ability to 
work together with Web Ontology languages such as DAML, OIL, and OWL. In fact, the highly modular J2EE architecture could easily accommodate the new extensions focused on agent interactions. As a result, Java or .NET based mobile agents would be able to interact with the semantically reach content - an important feature for e-commerce applications. The previously discussed eBay litigation illustrates the social sensitivity and complexity of issues involved, when using the mobile agents for e-commerce and other Web distributed applications.

As we mentioned, the rise of intelligent technologies only underscored the growing gap between the explosive growth of information technology and the slow pace of progress in software engineering. Intelligent agent technologies can become an important element in ongoing efforts to fill this gap. One suggested methodology, following the so called team-oriented software engineering paradigm, is to use agents themselves as software engineering tools. Michael N. Huns [8] emphasizes the key aspects of this approach:

- Level of abstraction - society

- Building block - agent

- Computational model - perceive, reason, act

- Design paradigm - cooperative interaction

- Architecture - managers, assistants, and peers

With the increasing use of the agents the new team-oriented (agent-oriented) paradigm may replace the 'old' software engineering methodologies. This may lead not only to the increasing use of agents in business, finance, and other applied areas but also to the use of agents "in enabling the rapid construction of robust and reusable software." Moreover, the agent based software development methodology would allow to "specify requirements, possibly in terms of social commitments and intentions." [10]. Of course, the old software engineering standards would have to be updated accordingly and the new standards may be developed.

It's instructive that the software engineering community already developed an unofficial "Robots Exclusion Protocol" on the Web [9]. The protocol is not mandatory for the guest-robots, and in the case of eBay the invading robots typically ignored the warning that they were not welcome (that's all the protocol allows to accomplish so far). Among the existing software engineering standards covering the major phases of the software development cycle, the possible candidates for agent-oriented updates are
IEEE Std 1229-1998, Std 830-1998, Std 1012-1998, Std 12207- 1996 and 1997 [10]. The efforts are also under way to develop the software engineering code of ethics. Needless to say, that in this respect the intelligent technologies deserve a special attention.

In this context, the social aspects of software engineering cannot be overestimated. The information technology professionals should be able to follow the mature and socially sensitive design methods reflected in the appropriate professional standards such as the Institute of Electrical and Electronics Engineers (IEEE) standards and recommendations.

The existing IEEE software engineering standards should address all the concerns related to security, privacy and the overall behavior of mobile intelligent agents by mandating some sort of security and agent negotiation mechanisms as an integral part of system architecture in general and of software system architecture in particular. Moreover, we urgently need a new set of standards related to the functionality of intelligent systems especially in respect to the agent-to-agent and agentto-human interactions.

Once the appropriate technical, social, and business constraints are incorporated into the standard software design procedures, thus becoming an integral constituent of the software engineering culture, they should be able to reshape both the educational environment and the software development practice. Consequently, the issues of legal liability could be also resolved in a constructive manner. In this respect, Johnson (2001) correctly emphasizes the importance of the Texas initiative to establish licenses for software engineers in the state of Texas (USA). Such a license should assume a high level of proficiency in the socially responsible software design methods, provided that the appropriate concerns are embedded into the standard design procedures. It also "helps the public to understand how to think about the products and services being provided by software engineers," and consequently enables the public to make moral choices about such products [11].

To achieve the aforementioned objectives, the software engineering community should focus on all the stages of software development process including the systems engineering, the software systems engineering, and the detailed software development. At every level, we should address the specific agent related issues as well as general concerns such as data privacy, data correctness, and the data collection techniques. The appropriate standards should be platform independent and addressed at each and every level of software engineering process (including systems analysis and design). 
The issues related to agent oriented distributed applications in electronic commerce are intrinsically linked to the semantic aspects of e-commerce environment. In the next section we consider how the emerging phenomenon of the Semantic Web may change the rules of the game.

\section{SEMANTIC WEB PROMISE}

The emergence of the Semantic Web presents special challenges related to agent-to-agent and agent-to-human interactions which involve the ability to handle metadata rich with a variety of social connotations. Among other issues, especially assuming that an agent may be required to make a decision dynamically, we have to consider the correlation between the ability to make a decision and the measure of responsibility for the decision taken - an important issue for mission-critical applications.

The Semantic Web term reflects the recognition of the fact that not only the amount of information available on the Web is growing at the tremendous speed, but also the relationships between the information sources become more and more complex. As a result, the traditional means to locate, retrieve, and evaluate information on the Web are no longer satisfactory. The Semantic Web concept assumes that explicit representation of data semantics (metadata) is the answer to information explosion we face; thus, the Semantic Web project is focused on the reshaping the Web into the complex semantic infrastructure; the essential elements of the infrastructure include (fig. 1 below shows a more detailed representation; see [12]):

- Web Ontologies representing different business domains in a semantically explicit way

- Agent enabled new markup languages, capable to work with Web Ontologies

- Ontology enabled security and trust infrastructure

\begin{tabular}{|c|c|c|}
\hline & Trust & $\mathbf{S}$ \\
\hline & Rules/Proof & e \\
\hline & Logic & $\mathbf{u}$ \\
\hline & Ontology & i \\
\hline & F/Schema & $\mathbf{t}$ \\
\hline XML & Namesnaces & $y$ \\
\hline URI & Innicode & \\
\hline
\end{tabular}

Fig. 1 - Semantic Web Infrastructure

The leading candidates for the future linguistic skeleton of the Semantic Web are XML based
RDF/S (Resource Description Framework/Schema), DAML (DARPA Agent Markup Language), OIL (Ontology Inference Layer), and OWL (Ontology Web Language). These languages can provide the long awaited framework for the semantically rich ecommerce transactions.

XML itself is instrumental in ensuring security of electronic transactions - XML compliant digital signatures represent one of the key elements of the electronic business infrastructure. At the same time, XML became the basis for a number of business oriented languages, such as Electronic Business XML (ebXML) developed by the United Nations Center for Trade Falicitation and Electronic Business; a group of Web services protocols such as Web Services Description Language (WSDL) are also based on XML.

The power of the Semantic Web would be lost without the ability to process metadata effectively and efficiently. The increasing power of knowledge representation requires new forms of distributed computing. Hence, the need to balance the new forms of metadata representation with the emerging paradigm of agent-oriented computing. The deep integration of the Semantic Web with intelligent agents in general and mobile agents in particular should provide a viable solution for distributed applications, allowing significantly to speed up the decision making processes and to reduce the overall network load.

On the whole, the integration of the Semantic Web, Web services, and agent technologies would make a significant impact on the development of efficient and scalable e-commerce applications. In fact, the DAML project has been focused on developing "a language aimed at representing semantic relations in machine-readable ways that will be compatible with current and future Internet technologies," in particular agent technologies [12].

As with many other advanced technologies (including Internet), the leading role in the Semantic $W e b$ project has been played by the US research agency DARPA engaged primarily in the military related research activities. At the same time, DARPA is looking into the ways to use intelligent agents for criminal detection and surveillance; using agents for monitoring the bio-terrorist attacks is only one example of law enforcement related application [13]. Among the most recent developments, we should mention DAML-S - an attempt to adjust DAML to Web services model [14]. Integration of DAML-S (or incorporating the similar features into OWL emerging as a new standard ontology language) with the existing business oriented Web services would further enhance the development of e-commerce applications. We should mention that 
DAML-S is already linked to the Web Services Description Language.

The more the agent technology is semantically enabled, the more autonomous can we expect the intelligent agents to be; such autonomy should translate into both technical as well as business benefits. Enabled to function in a heterogeneous ontology environment, the intelligent agents would allow the customers to personalize their e-commerce experience. Consequently, the agents could be entrusted with all or most of the details of typical ecommerce transactions. This includes all of the aforementioned stages of the buying process such as need identification, product brokering, negotiation etc.

We can expect that integration of the Semantic Web with agents and Web services will boost still lagging business-to-customer (B2C) e-commerce transactions. Currently, the fastest growing part of ecommerce is business-to-business (B2B) - likely "due ... to the fact that the vocabulary involved in business transactions is already codified for automated processing" [15]. Another key reason for the success of B2B e-commerce may involve the fact that B2B transactions have been already conducted for decades using established private networks with EDI (Electronic Data Interchange) data exchange. The development of Web-to-EDI and EDI-to-Web translators should further enhance B2B commerce by engaging smaller businesses into the global e-commerce chain.

The heterogeneous ontology environment on the Web could also enhance availability of agent-toagent Web interactions, leading eventually to the development of multiagent systems distributed over the global e-commerce network [6]. The multiagent systems may eventually require rethinking the whole Web architecture. Not only the details of the low level network architecture but also the local ontologies of the Semantic Web should be hidden from the users.

Generally speaking, the Web should be construed as ontology/knowledge independent distributed system. By this we mean that the users should be shielded from the heterogeneous details of the network architecture, the underlying operating systems, the structure of the data sources, and the local ontologies. This, in turn, could require the development of multilevel architecture with an elaborate system of mappings.

\section{CODA}

This paper was conceived as a brief survey of the opportunities and the challenges related to the application of intelligent agent technologies, the growing phenomena of the Semantic Web to the development of global e-commerce infrastructure. One of the key lessons learned is a necessity to focus research efforts on unifying the areas of intelligent software, Semantic Web, and Web services in order to fulfill the promise that information technology brought to the 'networked community' in general and to the business community in particular. In order to accomplish this objective, it's important to overcome the diversity between the different national and international standards in the area of Web services and the Semantic Web. This, in turn, requires the decisive shift in the existing software engineering paradigm at all levels - technical, legal, and educational.

\section{REFERENCES}

[1] P. Maes, R. H. Guttman \& A. G. Moukas. Agents that buy and sell, Communications of the ACM 42 (3) (1999, March). pp. 81-91.

[2] D. B. Lange \& M. Oshima. Dispatch your agents; shut off your machine, Communications of the ACM, 42(3) (1999, March ). pp. 88-89.

[3] T. Mandry, G. Pernul \& A. Rohm. Mobile agents on electronic markets, International Journal of Electronic Commerce, 5 (2) (2000). pp. 47-60.

[4] E. Turban, C. Wagner. Are intelligent ecommerce agents partners or predators?

Communications of the ACM 45 (5) (2002, May). pp. 84-90.

[5] BTP: Accessed online at 10/15/2003 at: (http://www.oasis-open.org/committees/businesstransactions/).

[6] K. M. McDermot Protecting Website Databases Through Intentional Tort or Contract: Ebay Meets UCITA. accessed online 06/10/2003 at: www.alliancelawgroup.com/news_article_11081.pdf

[7] M-P. Huget Extending Agent UML Protocol Diagrams. Technical Report ULCS02-014, Department of Computer Science, University of Liverpool, 2002. pp. 1-12. accessed online $6 / 10 / 2003$ $a t$ : http://www.dbgroup.unimo.it/IIA/references.html [8] M. N. Huns. Agent Teams: Building and Implementing Software. IEEE Internet Computing, 4 (1) (2000, January-February). pp. 93-95.

[9] Robots Exclusion Protocol: accessed online 06/10/2003

at:

http://www.robotstxt.org/wc/exclusion.htm

[10] IEEE Software engineering standards collection. 1-4. IEEE Press. Piscataway, N.J., 1999.

[11] D. Johnson. Computer ethics (3 ${ }^{\text {rd }}$ ed.). Prentice Hall. Upper Saddle River, NJ. 2001. pp. 66-67. 
[12] J. Hendler. Agents and the Semantic Web, IEEE Intelligent Systems, 16 (2) (2001, March/April). pp. 30-37.

[13] J. Mena. Investigative Data Mining for Security and Criminal Detection. Butterworth/Heinemann. New York, 2003.

[14] M. Paolucci, K. Sycara, and T. Kawamura. Delivering Semantic Web Services, Tech. report CMU-RI-TR-02-32, Robotics Institute, Carnegie Mellon University, May, 2003.

[15] D. Wong, N. Paciorek \& D. Moore. Javabased Mobile Agents. Communications of the ACM, 42 (3) (1999, March). pp. 92-102. 